\title{
Plasma palmitoylethanolamide (PEA) as a potential biomarker for impaired coronary function
}

Alessandra Quercioli ${ }^{1 \#}$, Federico Carbone ${ }^{2 \#}$, Aldo Bonaventura ${ }^{2}$, Luca Liberale ${ }^{2}$, Zoltan Pataky ${ }^{3}$, Aurélien Thomas ${ }^{4}$, Sébastien Lenglet ${ }^{4}$, Estelle Lauer ${ }^{4}$, Alain Golay ${ }^{3}$, Franco Dallegri $^{2,5}$, Vincenzo Di Marzo ${ }^{6}$, Thomas H. Schindler ${ }^{7,8^{*}}$, Fabrizio Montecucco ${ }^{2,5,9^{*}}$

${ }^{1}$ Division of Cardiology, "SS. Antonio e Biagio e Cesare Arrigo" Hospital, 6 via Venezia 16, 15121 Alessandria, Italy. ${ }^{2}$ First Clinic of Internal Medicine, Department of Internal Medicine, University of Genoa, 6 viale Benedetto XV, 16132 Genoa, Italy. ${ }^{3}$ Service of Therapeutic Education for Chronic Diseases, WHO Collaborating Centre, University Hospital of Geneva, University of Geneva, rue Gabrielle-Perret-Gentil 4, 1205 Geneva, Switzerland. ${ }^{4}$ Unit of Toxicology, CURML, Geneva-Lausanne, CHUV, HUG, rue Michel-Servet 1, 1211 Geneva, Switzerland. ${ }^{5}$ IRCCS AOU San Martino-IST, Genova, largo Benzi 1016143 Genoa, Italy. ${ }^{6}$ Endocannabinoid Research Group, Institute of Biomolecular Chemistry, Consiglio Nazionale delle Ricerche, Via Campi Flegrei 34, 80078 Pozzuoli, Naples, Italy. ${ }^{7}$ Division of Nuclear Medicine - Cardiovascular Section, Department of Radiology and Radiological Science, School of Medicine, Johns Hopkins University, JHOC 3225, 601 N. Caroline Street, Baltimore, MD 21287, USA. ${ }^{8}$ Division of Cardiology, Department of Medicine, Johns Hopkins University, Baltimore, MD, USA. ${ }^{9}$ Centre of Excellence for Biomedical Research (CEBR), University of Genoa, 9 viale Benedetto XV, 16132, Genoa, Italy. All author takes responsibility for all aspects of the reliability and freedom from bias of the data presented and their discussed interpretation.

Running title: PEA and coronary dysfunction 
Corresponding Author: Prof. Fabrizio Montecucco, MD, PhD, First Clinic of Internal Medicine, Department of Internal Medicine, University of Genoa, 6 viale Benedetto XV, 16132 Genoa, Italy, Tel: +39 01035386 94, Fax: +39 01035386 86, E-mail: Fabrizio.montecucco@unige.it

\section{Acknowledgements}

\# These authors equally contributed as first authors to this work. * These authors equally contributed as last authors to this work. This work was supported by Swiss National Science Foundation Grant to Prof. F. Montecucco (\#310030_152639/1) and to Prof. T. H. Schindler (\#3200B0-122237). 


\begin{abstract}
Background: Among endocannabinoid (EC)-related mediators, Oleoyl-ethanolamide (OEA) and Palmitoyl-ethanolamide (PEA), two endogenous PPAR $\alpha$ agonists with lipolytic and antiinflammatory action, respectively, are being actively investigated. Here, we assessed the potential association between plasma levels of PEA and OEA and coronary function in a cohort including normal, overweight, obese, and morbidly obese (MOB) individuals. Methods: Myocardial perfusion and endothelium-related myocardial blood flow (MBF) responses to cold pressor test (CPT) and during pharmacological vasodilation with dipyridamole were measured with ${ }^{13} \mathrm{~N}$-ammonia positron emission tomography/computed tomography. OEA and PEA were extracted from human plasma by liquid-liquid extraction, separated by liquid chromatography and quantified by mass spectrometry. Serum levels of intercellular adhesion molecule-1 and vascular cell adhesion molecule-1 (VCAM-1) were measured by colorimetric enzyme-linked immunosorbent assay. Results: Circulating levels of PEA and VCAM-1 were increased in MOB as compared to normal weight subjects. Circulating levels of OEA and PEA were associated with body mass index, but not with adhesion molecules. Increases of PEA levels were associated with and predictive of worsened coronary function in MOB and the overall cohort studied. Conclusion: Plasma levels of PEA are increased in MOB patients and associated with coronary dysfunction as a functional precursor of CAD process. Larger trials are needed to confirm PEA as a potential circulating biomarker of coronary dysfunction in both MOB patients and the general population.
\end{abstract}

Keywords: endocannabinoid system; OEA; PEA; coronary circulation; obesity; adhesion molecules. 


\section{Introduction}

Emerging evidence from both basic and clinical studies indicates that the endocannabinoid (EC) system might modulate critical pathophysiological processes in cardiovascular (CV) diseases, such as obesity, atherosclerosis, and their ischemic-related disorders in the heart and brain [1-4].

For instance, in obese $(\mathrm{OB})$ subjects without other $\mathrm{CV}$ risk factors, increased plasma levels of the EC Anandamide (AEA) negatively impacted on the coronary endothelial function [5]. Accordingly, gastric bypass-induced weight loss was associated with concomitant normalization of the coronary circulatory function and AEA plasma levels [6]. On the other hand, in a model of atherogenesis using mice deficient for fatty acid amide hydrolase (an enzyme degrading ECs), elevated circulating levels of ECs increased intraplaque neutrophil recruitment of inflammatory cells, thus potentially contributing to plaque vulnerability [7]. Related to the EC system, the AEA congeners, Oleoyl-ethanolamide (OEA) and Palmitoylethanolamide (PEA), are endogenous fatty acids produced by most mammalian cells, including human white subcutaneous adipocytes [8]. Unlike the ECs AEA and 2Arachydonoylglycerol (2-AG), PEA and OEA have no direct agonist activity at cannabinoid $\mathrm{CB}_{1}$ and $\mathrm{CB}_{2}$ receptors [9] and activate other molecular targets, including the peroxisome proliferator-activated receptor (PPAR)- $\alpha[10]$. The vascular properties of these compounds have been only partially investigated and remain unclear. PEA has also been proposed to potentiate AEA effects by inhibiting its metabolic degradation or by enhancing its affinity for the transient receptor potential vanilloid 1 channel (TRPV1) [11], whereas OEA can activate these channels directly [10]. This may explain findings from animal models, in which PEA and OEA were shown to induce vasorelaxation in small mesenteric arteries either by potentiating AEA activity at TRPV1 or per se via an unidentified $\mathrm{CB}_{2}$-like receptor [9]. 
On the basis of these premises, in this study we investigated the potential association between plasma levels of PEA and OEA and coronary function (endothelial-dependent and independent) in a human cohort including normal, overweight (OW), OB, and morbidly obese (MOB) subjects, all without other CV risk factors. Finally, the potential relationship between plasma levels of PEA and OEA and systemic endothelial activation markers, such as serum levels of intercellular adhesion molecule-1 (ICAM-1) and vascular cell adhesion molecule-1 (VCAM-1) was assessed.

\section{Methods}

\section{Study population and design}

The clinical characteristics of the different study groups were previously investigated and published [2]. In this sub-study, only study participants with normal stress-rest perfusion on ${ }^{13} \mathrm{~N}$-ammonia positron emission tomography (PET)/computed tomography (CT) images were included in the study analysis as such findings widely excluded the presence of obstructive coronary artery disease (CAD). All individuals recruited did not report arterial hypertension, smoking, diabetes mellitus, assumption of any cardiac or vasoactive medication, a history of variant angina, a family history of premature $\mathrm{CAD}$, or clinically manifested $\mathrm{CV}$ or any other systemic disease. In this sub-study, we were able to include 30 normal weight control subjects (CON) (body mass index [BMI]: 20 to $24.9 \mathrm{~kg} / \mathrm{m}^{2}$ ), $31 \mathrm{OW}$ (BMI: 25 to $29.9 \mathrm{~kg} / \mathrm{m}^{2}$ ), $21 \mathrm{OB}$ (BMI: 30.0 to $39.9 \mathrm{~kg} / \mathrm{m}^{2}$ ), and $25 \mathrm{MOB}$ (Table 1) [2]. Only 21 OB patients (on total $\mathrm{n}=25$ previously published) [2] were included based on availability for analysis of remaining serum samples. ${ }^{13} \mathrm{~N}$-ammonia PET/ CT measurements of myocardial blood flow (MBF) at rest and during vasomotor stress were performed in all patients at a fasting state to assess coronary circulatory function. The study was approved by the University Hospitals of Geneva Institutional Review Board (No. 07-183) and each participant signed the approved informed 
consent form [2]. The study protocol conforms to the ethical guidelines of the 1975 Declaration of Helsinki as reflected in a priori approval by the institution's human research committee.

\section{Assessment of myocardial flow with PET/CT}

Myocardial perfusion and $\mathrm{MBF}$, measured in $\mathrm{mL} / \mathrm{min} / \mathrm{g}$ of myocardial tissue, were determined with ${ }^{13} \mathrm{~N}$-ammonia PET/CT, serial PET image acquisition, and a two compartment tracer kinetic model as described previously [5]. Sympathetic stimulation with cold pressor test (CPT) was performed with the study participant immersing one hand in a slush of ice water. At $60 \mathrm{sec}$, approximately 500-550 MBq ${ }^{13} \mathrm{~N}$-ammonia was administered and serial PET images were acquired while immersion of the hand in ice water continued for at least another $60 \mathrm{sec}$. The predominantly endothelium-dependent CPT-induced flow response from rest was defined as the difference between rest and CPT induced MBF ( $\triangle \mathrm{MBF})$. Then, hyperemic MBF was induced with intravenous standard dose of $140 \mu \mathrm{g} / \mathrm{kg} / \mathrm{min}$ with dipyridamole over 4 minutes. After an additional 3 minutes at peak effect of pharmacologic vasodilation of arteriolar vessels, approximately 500-550 MBq ${ }^{13} \mathrm{~N}$-ammonia was injected intravenously again and acquisition of PET emission data was started. The stress PET scan was followed by a low dose CT scan for attenuation correction of the PET stress images during pharmacologic vasodilation. This was deemed necessary to account for possible changes in cardiac and pulmonary volumes owing to dipyridamole-induced hyperemia. Finally, the physical half-life (9.8 min) of ${ }^{13} \mathrm{~N}$-ammonia necessitated a time interval of about 45 minutes between repeat assessments of MBF with PET.

Heart rate, blood pressure, and a 12-lead electrocardiogram were recorded continuously during each MBF measurement. From the average of heart rate and systolic blood pressure during the first 2 min of each image acquisition, the rate-pressure product (RPP) was derived 
as an index of cardiac work. To account for possible inter-individual variations in coronary driving pressure, an index of global coronary vascular resistance was determined as the ratio of mean arterial blood pressure $(\mathrm{mmHg})$ to $\mathrm{MBF}(\mathrm{mL} / \mathrm{g} / \mathrm{min})$. In addition, $\mathrm{MBF}$ was normalized to the RPP, that is the myocardial work (averaged during the first 2 min of image acquisition; MBF divided by RPP multiplied by 10’000).

\section{Measurement of EC-like compounds}

OEA and PEA were extracted from $100 \mu \mathrm{L}$ of human plasma by liquid-liquid extraction and separated by liquid chromatography (Ultimate 3000 RS, Dionex, CA, USA). Quantitative analyses were performed on a 5500 QTrap® triple quadrupole/linear ion trap (QqQLIT) mass spectrometer equipped with a TurboIon-SprayTM interface (AB Sciex, Concord, ON, Canada) [12].

\section{Detection of serum levels of soluble adhesion molecules}

Colorimetric enzyme-linked immunosorbent assay from kits to measure serum levels of ICAM-1 and VCAM-1 (R\&D Systems Inc. Minneapolis, MN, USA) were used following manufacturer instructions. The limit of detection was $15.625 \mathrm{pg} / \mathrm{ml}$ for ICAM-1 and 15.625 $\mathrm{pg} / \mathrm{ml}$ for VCAM-1. Mean intra- and inter-assay coefficients of variation were below $6 \%$.

\section{Statistical analysis}

Statistical analyses were performed with IBM SPSS Statistics for Windows, version 20.0 (Armonk, NY: IBM Corp.). Continuous variables were expressed as median and interquartile range (IQR), given their non-parametric distribution. Comparisons between groups were drawn by nonparametric Mann-Whitney U-test. Spearman rank test was performed to establish correlations between EC-like compounds and MBF during hyperemia and $\triangle \mathrm{MBF}$ to 
CPT in overall cohort and in MOB. The accuracy of PEA to predict MBF during hyperemia and $\triangle \mathrm{MBF}$ to $\mathrm{CPT}$ in the overall cohort was assessed by the receiver operating characteristic (ROC) analysis. The area under the curve (AUC) was given with 95\% confidence interval (CI) obtained using MedCalc 12.5 (MedCalc Software, Ostend, Belgium), and the best cut-off point of PEA was calculated by maximizing sensitivity according to the Youden index. Finally, multivariate risk analysis was performed by linear and logistic regression models, respectively presented as $\beta$ coefficient and odds ratio (OR) with corresponding $95 \% \mathrm{CI}$. In the linear regression models in both overall cohort and MOB, PEA was consecutively set as the independent variable whereas age, gender, BMI, ICAM-1, and VCAM-1 as well as MBF during hyperemia and $\triangle \mathrm{MBF}$ were set as the dependent variables. In the logistic regression models in the overall cohort, categorized PEA (according to the cut-off values) was set as the independent variable, whereas MBF during hyperemia and $\triangle \mathrm{MBF}$ were set as the dependent variables. All test procedures were two-tailed, and $\mathrm{p} \leq 0.05$ was considered statistically significant.

\section{Results}

\section{Circulating levels of PEA and VCAM-1 are increased in $M O B$}

The clinical and anthropometrical characteristics, the fat amount, the metabolic profile, and the hemodynamic and myocardial blood flow parameters in the different study groups (classified on BMI values) were reported in a previously published study [2]. In Table 1, the circulating levels of PEA and OEA as well as of adhesion molecules (possibly related with endothelial dysfunction/activation) are shown. Circulating levels of PEA and VCAM-1 were significantly increased in MOB patients as compared to CON. On the other hand, no changes in these parameters were found in $\mathrm{OB}$ and $\mathrm{OW}$ patients as compared to $\mathrm{CON}$ (Table 1). In addition, a small decrease in OEA levels was shown in OW, but not in OB and MOB as 
compared with CON (Table 1). Finally, ICAM-1 levels in OW, OB, and MOB were comparable to CON (Table 1).

Circulating levels of AEA-like compounds are associated with BMI, but not adhesion molecules.

In order to investigate the potential independent association between circulating levels of AEA-like compounds and age, gender, BMI, and adhesion molecules, we used linear regression with univariate and multivariate models in the entire study cohort. Circulating levels of PEA were positively associated with BMI $(\beta=0.304, p=0.002)$ (Table 2). This association remained statistically significant after the adjustment for age, gender, ICAM-1, and VCAM-1 levels $(\beta=0.350, \mathrm{p}=0.001)$ (Table 2$)$. OEA was differently associated with BMI and VCAM-1 $(\beta=0.268, p=0.010$ and $\beta=-0.241, p=0.017$, respectively $)$ only when using a multivariate analysis (Table 2).

High circulating levels of PEA, but not OEA, are associated with and predict parameters of coronary dysfunction.

Circulating levels of PEA were inversely correlated with MBF during hyperemia (a parameter of endothelium-independent coronary vasodilation) and $\triangle \mathrm{MBF}$ to $\mathrm{CPT}$ (a parameter of endothelium-dependent coronary vasodilation) (Supplementary figure 1A and 1B) in the entire study cohort. This inverse association was confirmed by a linear regression model, showing that circulating levels of PEA were inversely associated with coronary vasodilation using univariate and multivariate analysis (Table 3). No significant association was demonstrated between OEA levels and MBF during hyperemia or $\triangle \mathrm{MBF}$ to $\mathrm{CPT}$ in the entire study cohort (Supplementary figure 1C and 1D and Table 3). Particularly in MOB patients, PEA, but not OEA, levels were also found to be inversely and strongly correlated with 
coronary vasodilation parameters (PEA vs. MBF during hyperemia: $r=-0.717, \mathrm{p}<0.001$; PEA vs. $\triangle \mathrm{MBF}$ to CPT: $\mathrm{r}=-0.537, \mathrm{p}<0.007$ ) (Supplementary figure $2 \mathrm{~A}-2 \mathrm{D}$ ). ROC analysis showed that PEA levels had a significant prognostic accuracy to predict MBF during hyperemia or $\triangle \mathrm{MBF}$ to $\mathrm{CPT}$. Indeed, according to the Youden index, a PEA value $\leq 3.176 \mathrm{ng} / \mathrm{mL}$ predicted high MBF during hyperemia (AUC: 0.695, $\mathrm{p}=0.002$ ), while a PEA value $\leq 2.465 \mathrm{ng} / \mathrm{mL}$ predicted high $\triangle \mathrm{MBF}$ to $\mathrm{CPT}$ (AUC: $0.642, \mathrm{p}=0.018$ ) (Supplementary figure $3 \mathrm{~A}$ and $3 \mathrm{~B}$ ). Logistic regression indicated that a PEA value $\leq 3.176 \mathrm{ng} / \mathrm{mL}$ is a predictor of best $\mathrm{MBF}$ during hyperemia at univariate and multivariate analyses (independently of age, gender and BMI) (Table 4). As potentially expected from previous association with BMI, PEA levels also predicted BMI (Table 4). Considering endothelium-dependent coronary vasodilation, PEA levels value $\leq 2.465 \mathrm{ng} / \mathrm{ml}$ predicted $\triangle \mathrm{MBF}$ to $\mathrm{CPT}$ values only with univariate analysis, but not independently of potential confounders (such as age, gender, and BMI). These results strongly support that high circulating levels of PEA are associated with coronary dysfunction in a general apparently healthy population (the whole study cohort) and in MOB subjects.

\section{Discussion}

The main findings of this observational clinical research article are represented by the increase of plasma levels of PEA in MOB patients as compared to normal controls and by the fact that high circulating levels of PEA are associated and predictive of coronary dysfunction both in MOB patients and in the overall study cohort. Functional and structural alterations of the coronary microcirculation are deemed responsible for impairment of myocardial perfusion and ischemia usually referred to as coronary microvascular dysfunction (CMD) [13]. As CMD can alter the normal physiology of coronary vessels, these changes lead to an impaired capacity of MBF to respond to different myocardial oxygen demands. CMD has been found to be sustained by various functional mechanisms leading either to altered dilation or 
augmented constriction of coronary microvessels. In particular, impaired vasodilation can result from changes in endothelium-dependent mechanisms associated with metabolic diseases, such as obesity [13].

Our research group previously demonstrated that levels of PEA and OEA were altered in the aorta and the visceral adipose tissue of atherosclerotic mice [14]. Nevertheless, the circulating levels of ECs were suggested to be influenced by the body weight [15]. On the other hand, high body weight was independently associated with coronary dysfunction ranging from alterations in endothelium-related coronary vasomotion in OW subjects to a modification of the entire vasodilator capacity in OB subjects [16].

In the present sub-study, when considering the overall cohort, the circulating levels of PEA were inversely correlated with coronary function parameters. Interestingly, PEA levels were also found to predict coronary dysfunction. The predictive value of PEA was even more relevant when targeting MOB patients. Importantly, the association between PEA and coronary parameters were independent of BMI, suggesting PEA levels as a promising biomarker of coronary dysfunction in subjects without a known CAD. On the other hand, PEA levels failed to be associated with other circulating biomarkers of endothelial dysfunction/activation, such as soluble ICAM-1 and VCAM-1. Although controversial, circulating levels of adhesion molecules were previously described as generally related with endothelial injury and CV risk [17-19]. However, the modification of these parameters were more related to a "non-specific" endothelial dysfunction in different arterial districts instead of being selective for certain key organs, such as the heart, the brain, and the kidney. Although PEA was not correlated with endothelial markers, we were able to show that PEA levels displayed an inverse correlation with $\triangle \mathrm{MBF}$ to $\mathrm{CPT}$, especially in MOB patients. In particular, PEA levels value $\leq 2.465 \mathrm{ng} / \mathrm{mL}$ predicted a better endothelium-dependent 
coronary vasodilation. Therefore, PEA levels were selectively associated with a coronary endothelial dysfunction instead of systemic endothelial alteration.

The correlative nature of the present study does not allow us to speculate as to whether PEA plays a causative role for, or represents a protective adaptive mechanism against coronary dysfunction. The previously reported vasodilatory and anti-inflammatory actions of this mediator [20, 21], the high safety reported following chronic treatment with synthetic PEA in several clinical studies [22-24], and its lack of CV side effects strongly argue in favor of the latter hypothesis as well as the benign CV profile of its major target, PPAR- $\alpha$. Indeed, several pathological conditions exist in either human subjects or animal models in which exogenous PEA is known to produce a therapeutic action and local concentrations of endogenous PEA are found to be elevated $[25,26]$.

\section{Study limitations}

Since the current study was not planned as prospective, we were not able to predict the nature of the association between high plasma PEA levels and the risk of CV events. The study has also another limitation in the number of patients enrolled, which was small, so that our conclusions need confirmatory larger studies. The present work should therefore be considered as an observational study comparing samples collected in a definite time without considering peptide variations before and after weight loss.

\section{Conclusion}

Plasma PEA levels might represent a potential biomarker of coronary dysfunction in a cohort of subjects without $\mathrm{CAD}$ and especially in MOB patients. Additional larger trials are needed to confirm PEA as a potential circulating biomarker of coronary dysfunction in both MOB patients and in general population. 


\section{References}

[1] Montecucco F, Lenglet S, Quercioli A, Burger F, Thomas A, Lauer E, et al. Gastric bypass in morbid obese patients is associated with reduction in adipose tissue inflammation via N-oleoylethanolamide (OEA)-mediated pathways. Thromb Haemost. 2015;113:838-50.

[2] Quercioli A, Pataky Z, Montecucco F, Carballo S, Thomas A, Staub C, et al. Coronary vasomotor control in obesity and morbid obesity: contrasting flow responses with endocannabinoids, leptin, and inflammation. JACC Cardiovasc Imaging. 2012;5:805-15.

[3] Montecucco F, Di Marzo V, da Silva RF, Vuilleumier N, Capettini L, Lenglet S, et al. The activation of the cannabinoid receptor type 2 reduces neutrophilic protease-mediated vulnerability in atherosclerotic plaques. Eur Heart J. 2012;33:846-56.

[4] Montecucco F, Di Marzo V. At the heart of the matter: the endocannabinoid system in cardiovascular function and dysfunction. Trends Pharmacol Sci. 2012;33:331-40.

[5] Quercioli A, Pataky Z, Vincenti G, Makoundou V, Di Marzo V, Montecucco F, et al. Elevated endocannabinoid plasma levels are associated with coronary circulatory dysfunction in obesity. Eur Heart J. 2011;32:1369-78.

[6] Quercioli A, Montecucco F, Pataky Z, Thomas A, Ambrosio G, Staub C, et al. Improvement in coronary circulatory function in morbidly obese individuals after gastric bypass-induced weight loss: relation to alterations in endocannabinoids and adipocytokines. Eur Heart J. 2013;34:2063-73.

[7] Lenglet S, Thomas A, Soehnlein O, Montecucco F, Burger F, Pelli G, et al. Fatty acid amide hydrolase deficiency enhances intraplaque neutrophil recruitment in atherosclerotic mice. Arterioscler Thromb Vasc Biol. 2013;33:215-23.

[8] Gonthier MP, Hoareau L, Festy F, Matias I, Valenti M, Bes-Houtmann S, et al. Identification of endocannabinoids and related compounds in human fat cells. Obesity (Silver Spring). 2007;15:837-45. 
[9] Ho WS, Barrett DA, Randall MD. 'Entourage' effects of N-palmitoylethanolamide and Noleoylethanolamide on vasorelaxation to anandamide occur through TRPV1 receptors. $\mathrm{Br} \mathrm{J}$ Pharmacol. 2008;155:837-46.

[10] Karwad MA, Macpherson T, Wang B, Theophilidou E, Sarmad S, Barrett DA, et al. Oleoylethanolamine and palmitoylethanolamine modulate intestinal permeability in vitro via TRPV1 and PPARalpha. FASEB J. 2016.

[11] Re G, Barbero R, Miolo A, Di Marzo V. Palmitoylethanolamide, endocannabinoids and related cannabimimetic compounds in protection against tissue inflammation and pain: potential use in companion animals. Vet J. 2007;173:21-30.

[12] Thomas A, Hopfgartner G, Giroud C, Staub C. Quantitative and qualitative profiling of endocannabinoids in human plasma using a triple quadrupole linear ion trap mass spectrometer with liquid chromatography. Rapid Commun Mass Spectrom. 2009;23:629-38.

[13] Camici PG, d'Amati G, Rimoldi O. Coronary microvascular dysfunction: mechanisms and functional assessment. Nat Rev Cardiol. 2015;12:48-62.

[14] Montecucco F, Matias I, Lenglet S, Petrosino S, Burger F, Pelli G, et al. Regulation and possible role of endocannabinoids and related mediators in hypercholesterolemic mice with atherosclerosis. Atherosclerosis. 2009;205:433-41.

[15] Rubio MA, Gargallo M, Isabel Millan A, Moreno B. Drugs in the treatment of obesity: sibutramine, orlistat and rimonabant. Public Health Nutr. 2007;10:1200-5.

[16] Schindler TH, Cardenas J, Prior JO, Facta AD, Kreissl MC, Zhang XL, et al. Relationship between increasing body weight, insulin resistance, inflammation, adipocytokine leptin, and coronary circulatory function. J Am Coll Cardiol. 2006;47:1188-95.

[17] Park M, Kulkarni A, Beatty A, Ganz P, Regan M, Vittinghoff E, et al. Soluble endothelial cell selective adhesion molecule and cardiovascular outcomes in patients with 
stable coronary disease: A report from the Heart and Soul Study. Atherosclerosis. 2015;243:546-52.

[18] Poredos P, Jezovnik MK. The Role of Inflammatory Biomarkers in the Detection and Therapy of Atherosclerotic Disease. Curr Vasc Pharmacol. 2016.

[19] Schmidt EP, Kuebler WM, Lee WL, Downey GP. Adhesion Molecules: Master Controllers of the Circulatory System. Compr Physiol. 2016;6:945-73.

[20] Impellizzeri D, Esposito E, Attley J, Cuzzocrea S. Targeting inflammation: new therapeutic approaches in chronic kidney disease (CKD). Pharmacol Res. 2014;81:91-102.

[21] Skaper SD, Facci L, Barbierato M, Zusso M, Bruschetta G, Impellizzeri D, et al. NPalmitoylethanolamine and Neuroinflammation: a Novel Therapeutic Strategy of Resolution. Mol Neurobiol. 2015;52:1034-42.

[22] Chirchiglia D, Della Torre A, Signorelli F, Volpentesta G, Guzzi G, Stroscio CA, et al. Administration of palmitoylethanolamide in combination with topiramate in the preventive treatment of nummular headache. Int Med Case Rep J. 2016;9:193-5.

[23] Di Paola R, Impellizzeri D, Fusco R, Cordaro M, Siracusa R, Crupi R, et al. Ultramicronized palmitoylethanolamide $(\mathrm{PEA}-\mathrm{um}((\mathrm{R})))$ in the treatment of idiopathic pulmonary fibrosis. Pharmacol Res. 2016;111:405-12.

[24] Andresen SR, Bing J, Hansen RM, Biering-Sorensen F, Johannesen IL, Hagen EM, et al. Ultramicronized palmitoylethanolamide in spinal cord injury neuropathic pain: a randomized, double-blind, placebo-controlled trial. Pain. 2016;157:2097-103.

[25] Iannotti FA, Di Marzo V, Petrosino S. Endocannabinoids and endocannabinoid-related mediators: Targets, metabolism and role in neurological disorders. Prog Lipid Res. 2016;62:107-28.

[26] Petrosino S, Di Marzo V. The pharmacology of palmitoylethanolamide and first data on the therapeutic efficacy of some of its new formulations. Br J Pharmacol. 2016. 
Table 1. Levels of endocannabinoid-like compounds and adhesion molecules in the study groups ( $n=107)$.

\begin{tabular}{|c|c|c|c|c|c|c|c|}
\hline & $\begin{array}{c}\text { CON } \\
(n=30)\end{array}$ & $\begin{array}{c}\text { OW } \\
(n=31)\end{array}$ & $\begin{array}{l}p \text {-value } \\
v s . \text { CON }\end{array}$ & $\begin{array}{c}\text { OB } \\
(n=21)\end{array}$ & $\begin{array}{l}p \text {-value } \\
\text { vs. CON }\end{array}$ & $\begin{array}{c}\text { MOB } \\
(n=25)\end{array}$ & $\begin{array}{l}p \text {-value } \\
\text { vs. CON }\end{array}$ \\
\hline $\mathrm{PEA}, \mathrm{ng} / \mathrm{ml}$ & $2.62(2.36-3.01)$ & $2.47(2.07-2.96)$ & 0.184 & $2.67(2.23-3.19)$ & 0.953 & $3.07(2.54-3.47)$ & 0.016 \\
\hline OEA, ng/ml & $2.64(2.32-2.90)$ & $2.05(1.80-2.83)$ & 0.022 & $2.45(2.26-2.66)$ & 0.143 & $2.79(2.35-3.19)$ & 0.288 \\
\hline ICAM-1, ng/ml & $145.18(75.93-206.57)$ & $119.65(75.13-161.83)$ & 0.145 & $121.70(75.00-188.89)$ & 0.428 & $185.12(126.70-247.45)$ & 0.141 \\
\hline VCAM-1, ng/ml & $183.51(129.30-315.72)$ & $188.51(72.82-254.94)$ & 0.526 & $261.64(117.21-380.57)$ & 0.417 & $325.08(205.04-425.54)$ & 0.011 \\
\hline
\end{tabular}

Values are expressed as median (IQR). CON, controls; OW, overweight; OB, obesity; MOB, morbidly obesity; PEA, N-Palmitoyl-ethanolamide; OEA, N-Oleoylethanolamide. $p$-values vs. CON (Mann Whitney U-test). 
Table 2. Linear regression showing the relationship of the endocannabinoids (PEA and OEA) with the biomarkers of endothelial dysfunction ICAM-1 and VCAM-1.

\begin{tabular}{lcccccccc}
\hline & \multicolumn{4}{c}{ PEA $(\mathbf{n g} / \mathbf{m l})$} & \multicolumn{4}{c}{ OEA $(\mathbf{n g} / \mathbf{m l})$} \\
\cline { 2 - 9 } & \multicolumn{2}{c}{ Univariate } & \multicolumn{1}{c}{ Multivariate } & \multicolumn{2}{c}{ Univariate } & \multicolumn{1}{c}{ Multivariate } \\
\cline { 2 - 9 } & $\boldsymbol{\beta}$ & $\boldsymbol{p}$-value & $\boldsymbol{\beta}$ & $\boldsymbol{p}$-value & $\boldsymbol{\beta}$ & $\boldsymbol{p}$-value & $\boldsymbol{\beta}$ & $\boldsymbol{p}$-value \\
\hline Age, years & 0.012 & 0.903 & -0.042 & 0.659 & -0.086 & 0.381 & -0.110 & 0.256 \\
Gender, male & 0.030 & 0.758 & 0.089 & 0.354 & -0.069 & 0.482 & -0.005 & 0.980 \\
BMI, $\mathrm{kg} / \mathrm{m}^{2}$ & 0.304 & $\mathbf{0 . 0 0 2}$ & 0.350 & $\mathbf{0 . 0 0 1}$ & 0.180 & 0.066 & 0.268 & $\mathbf{0 . 0 1 0}$ \\
ICAM-1, ng/ml & -0.178 & 0.069 & -0.167 & 0.079 & -0.169 & 0.085 & -0.147 & 0.124 \\
VCAM-1, ng/ml & -0.011 & 0.911 & -0.122 & 0.219 & -0.164 & 0.094 & -0.241 & $\mathbf{0 . 0 1 7}$ \\
\hline
\end{tabular}

PEA: N-Palmitoyl-ethanolamide; OEA: N-Oleoyl-ethanolamide; BMI: body mass index; CI: confidence interval; ICAM: intracellular adhesion molecule; ICAM: intracellular adhesion molecule; VCAM: vascular cell adhesion molecule 
Table 3. Univariate linear regression showing the relationship of the endocannabinoids (PEA and

\begin{tabular}{|c|c|c|c|c|c|c|c|c|}
\hline & \multicolumn{4}{|c|}{ PEA (ng/ml) } & \multicolumn{4}{|c|}{ OEA (ng/ml) } \\
\hline & \multicolumn{2}{|c|}{$\begin{array}{l}\text { Overall cohort } \\
(n=107)\end{array}$} & \multicolumn{2}{|c|}{$\begin{array}{l}\text { MOB } \\
(n=25)\end{array}$} & \multicolumn{2}{|c|}{$\begin{array}{l}\text { Overall cohort } \\
(n=107)\end{array}$} & \multicolumn{2}{|c|}{$\begin{array}{c}\text { MOB } \\
(n=25)\end{array}$} \\
\hline & $\beta$ & $p$-value & $\beta$ & $p$-value & $\beta$ & $p$-value & $\beta$ & $p$-value \\
\hline $\begin{array}{l}\text { MBF during } \\
\text { hyperemia }\end{array}$ & -0.261 & 0.008 & -0.614 & 0.001 & -0.110 & 0.271 & -0.242 & 0.254 \\
\hline$\triangle \mathrm{MBF}$ to $\mathrm{CPT}$ & -0.234 & 0.016 & -0.419 & 0.041 & -0.162 & 0.098 & -0.316 & 0.133 \\
\hline
\end{tabular}

OEA) with the coronary function.

PEA: N-Palmitoyl-ethanolamide; OEA: N-Oleoylethanolamide; $\mathrm{MOB}$ : morbidly obese; MBF: myocardial blood flow; CPT: cold pressor testing; 
Table 4. Logistic regression showing the risk worse endothelial function according serum categorized levels of PEA.

\begin{tabular}{|c|c|c|c|c|c|c|}
\hline & \multicolumn{3}{|c|}{ Univariate model } & \multicolumn{3}{|c|}{ Multivariate model } \\
\hline & OR & $95 \% \mathrm{CI}$ & $p$-value & OR & $95 \% \mathrm{CI}$ & $p$-value \\
\hline \multicolumn{7}{|l|}{$\begin{array}{l}\text { PEA } \\
\text { (cutoff } \leq 3.176 \\
\text { ng/ml) }\end{array}$} \\
\hline $\begin{array}{l}\text { MBF during } \\
\text { hyperemia }\end{array}$ & 0.23 & $0.07-0.66$ & 0.006 & 0.26 & $0.08-0.85$ & 0.026 \\
\hline Age & 1.01 & $0.98-1.06$ & 0.377 & 0.99 & $0.95-1.04$ & 0.640 \\
\hline Gender, male & 1.94 & $073-5.19$ & 0.186 & 1.95 & $0.65-5.83$ & 0.232 \\
\hline BMI & 1.06 & $1.01-1.11$ & 0.021 & 1.06 & $1.01-1.12$ & 0.035 \\
\hline \multicolumn{7}{|l|}{$\begin{array}{l}\text { PEA } \\
\text { (cutoff } \leq 2.465 \\
\text { ng/ml) }\end{array}$} \\
\hline$\triangle \mathrm{MBF}$ to $\mathrm{CPT}$ & 0.05 & $0.01-0.61$ & 0.018 & 0.11 & $0.01-2.19$ & 0.149 \\
\hline Age & 1.00 & $0.96-1.03$ & 0.894 & 0.99 & 0.99-1.02 & 0.985 \\
\hline Gender, male & 1.28 & $0.57-2.86$ & 0.553 & 1.14 & $0.46-2.82$ & 0.771 \\
\hline BMI & 1.06 & $1.01-1.11$ & 0.032 & 1.04 & $0.98-1.01$ & 0.220 \\
\hline
\end{tabular}

OR: odds ratio; CI: confidence interval; PEA: N-Palmitoyl-ethanolamide; MBF: myocardial blood flow; CPT: cold pressor testing; 